\title{
Vehicle security system using ARM controller
}

\author{
Prof. Ajay Wadhawe ${ }^{1}$, Ganesh Yadav ${ }^{2}$

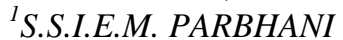 \\ ${ }^{2}$ S.S.I.E.M. PARBHANI
}

\begin{abstract}
Road accidents are a human tragedy. This involves high human suffering and pecuniary costs in terms of untimely sudden death, injuries and loss of inherent income. In this paper, a system is proposed where the main objective is to detect road signs from a moving vehicle and also enables intelligent detection of an accident at any place and reports about the accident on predefined numbers of dear one. The system will use one signal transmitter in each and every symbol or message board at road side and whenever any vehicle passes from that symbol the receiver situated inside the vehicle i.e. In-Car System will receive the signals and display proper message or the symbol details on display connected in car. Road Traffic Sign Detection is a technology by which a vehicle is able to recognize the traffic signs which are on the road e.g. "speed limit" or "school" or "turn ahead". This infrastructure is expected to deliver multiple road safety and driving assistance applications.
\end{abstract}

Keywords: ARM7, VANET,GPS Modem, RF module, Vibration sensor, GSM Modem.

\section{Introduction}

With increase in population it increases of the vehicles on road, safety and traffic jam have become three tremendous problems. Road traffic injuries (RTIs) and casualty have emerged as a major public health concern, with RTIs having become one of the leading causes of deaths, disabilities and hospitalizations which impose severe socio-economic costs across the world. Chained accidents in highway result in numerous losses of lives and properties. On the other hand, sometimes a minor accident in downtown triggers traffic congestion, this situation is much worse in metro city during the heavy traffic [1]. World Health Statistics 2008 cited in Global Status Report on Road Safety states that RTIs in 2004 were the 9th leading cause of death and at current rates by 2030 are expected to be the 5th leading cause of death, overtaking diabetes and HIV / AIDS [2]. Car accidents are one of the leading causes of death in present days in India. In 2005 near about 1 lakh of deaths resulted from 4.4 lakh of traffic accidents. In 2008 this figure reaches to 1.2 lakh of death and in 2010 death toll is near about 1.36 lakh for a 5 lakh accidents [2]. Hence the United Nations has rightly proclaimed 2011-20 as the decade of action on road safety and have called upon all member countries to prepare a decadal action plan for implementation in their respective countries so that the present rising trend of road accidents stabilizes and is reversed by the year 2020. Moreover, survey shows that each minute that an injured crash victim does not receive emergency medical care can make a large difference in their survival rate, e.g., analysis shows that reducing accident response time by one minute correlates to a six percent difference in the number of lives saved. An effective approach for reducing traffic fatalities, therefore, is to reduce the time between when an accident occurs and when first responders, such as medical personnel, are dispatched to the scene of the accident.

Traffic accidents. In 2008 this figure reaches to 1.2 lakh of death and in 2010 death toll is near about 1.36 lakh for a 5 lakh accidents [2]. Hence the United Nations has rightly proclaimed 2011-20 as the decade of action on road safety and have called upon all member countries to prepare a decadal action plan for implementation in their respective countries so that the present rising trend of road accidents stabilizes and is reversed by the year 2020. Moreover, survey shows that each minute that an injured crash victim does not receive emergency medical care can make a large difference in their survival rate, e.g., analysis shows that reducing accident response time by one minute correlates to a six percent difference in the number of lives saved.. An effective approach for reducing traffic fatalities, therefore, is to reduce the time between when an accident occurs and when first responders, such as medical personnel, are dispatched to the scene of the accident. The current scenario of road sign boards is shown in fig.1. These pictures state the problems which are faced by a driver while driving the vehicle. These problems derive due to various reasons as shown in fig. 1 . Here some symbols are rusted, partially collapsed or hidden in the bushes. 


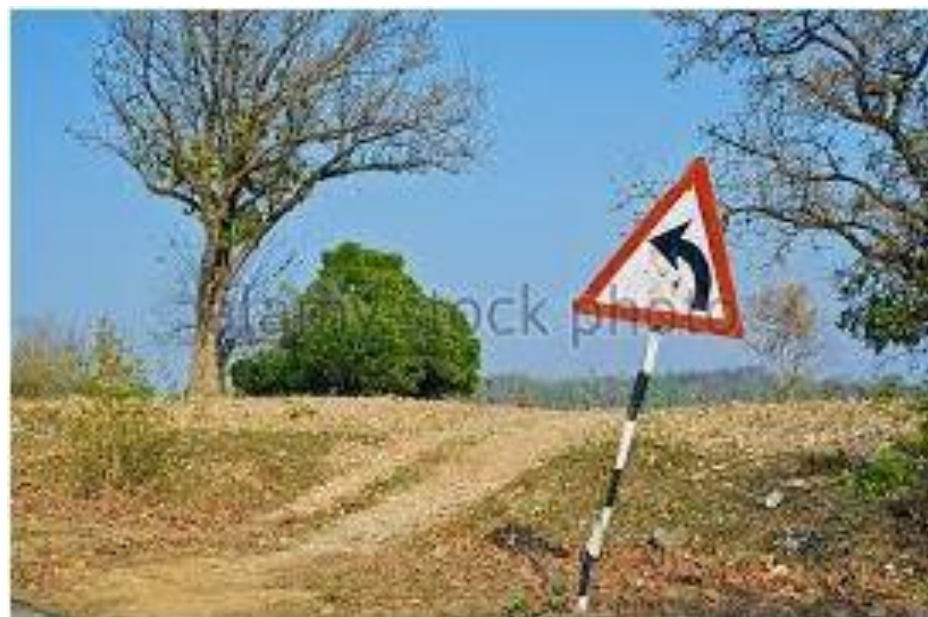

Figure 1: Current Scenario So, to help the driver we are implementing this system. Generally road signs consist of three properties; firstly they are represented by colors such as Red, Blue and Brown etc. Secondly they consists of a particular outlying shape such as Circular, Triangular, Octagonal, Square etc. The inner contents of the roadside symbol represent the third property, which may fluctuate depending on the application of the road side symbol. The challenge is to process queries in this highly mobile distributed database stored at fixed sites that is updated with an acceptable delay, overhead and accuracy. The main aim of this system is to alert the driver about the upcoming symbol. The system prevents future accidents that are likely to occur.

\section{Related Work}

Literature review should include current thinking, findings, and approaches to the problem.

Bankar Sanket Anil[1], this paper presents a system which gives an idea about what can be done to provide medical help and other facilities after accident as soon as possible. Accident can be detected using flex sensor and accelerometer, while location of accident will be informed to desired persons such as nearest hospital, police and owner of vehicle through sms sent using GSM modem containing co-ordinates obtained from GPS along with time of accident and vehicle number. Camera located inside vehicle will transmit real time video to see current situation of passengers inside vehicle.

Montaser N. Ramadan et al. [2] This system is implemented for anti-theft using an embedded system occupied with a Global Positioning System and a Global System of Mobile. The client interacts through this system with vehicles and determines their current locations and status using Google Earth. The user can track the position of targeted vehicles on Google Earth. Using GPS locator, the target current location is determined and sent, along with various parameters received by vehicle's data port, via Short Message Service (SMS) through GSM networks to a GSM modem that is connected to computer or laptop. The GPS coordinates are corrected using a discrete Kalman filter. In this paper, a low-cost vehicle tracking and monitoring system is presented.

Pravada P. Wankhade 3. This project deals with the design \& development of a theft control system for an automobile, which is being used to prevent/control the theft of a vehicle. The developed system makes use of an embedded system based on Global System for Mobile communication (GSM) technology. The designed \& developed system is installed in the vehicle. An interfacing mobile is also connected to the microcontroller, which is in turn, connected to the engine. Once, the vehicle is being stolen, the information is being used by the vehicle owner for further processing. The information is passed onto the central processing insurance system which is in the form of the sms, the microcontroller unit reads the sms and sends it to the Global Positioning System (GPS) module and using the triangulation method, GPS module feeds the exact location in the form of latitude and longitude to the user's mobile. By reading the signals received by the mobile, one can control the ignition of the engine; say to lock it or to stop the engine immediately. The main concept in this design is introducing the mobile communication into an embedded system. The designed unit is very simple $\&$ low cost. The entire designed unit is on a single chip.

Pranav Dhole 4. Smart Accident Detection \& Assistance System, Automatically detects accident of the vehicle. It also Finds the exact location of the vehicle Informs the central server about the accident and notifies the assistance services automatically.

Vidya Lakshmi 5. An automatic alarm device for traffic accidents is introduced in this paper. It can automatically find a traffic accident, search for the spot and then send the basic information to first aid center within two seconds covering geographical coordinates, the time and circumstances in which a traffic accident 
takes place. GPS software is fitted in the vehicle will now start communicate with the satellite and get the latitude and longitude values and send the information to the centralized server.

Then the server will search the nearest hospital and send the accident information to the hospital. The hospital will then be sending the ambulance to the accident zone. Then the injured people will be saved as soon as possible. This process will save time in particular for the areas in the outer part of main zone.

NOOPUR PATNE 6, In recent years many researcher are working on VANET and trying to implement the concepts in real world. Vehicular ad hoc networks (VANETs) are being developed to provide ondemand wireless communication infrastructure among vehicles and authorities. Such an infrastructure is expected to deliver multiple road safety and driving assistance applications. In this paper, a system is proposed where the main objective is to detect road signs from a moving vehicle. The system will use one signal transmitter in each and every symbol or message board at road side and whenever any vehicle passes from that symbol the receiver situated inside the vehicle i.e. In-Car System will receive the signals and display proper message or the symbol details on display connected in car. Road Traffic Sign Detection is a technology by which a vehicle is able to recognize the traffic signs which are on the road e.g. "speed limit" or "school" or "turn ahead".

\section{Block Diagram}
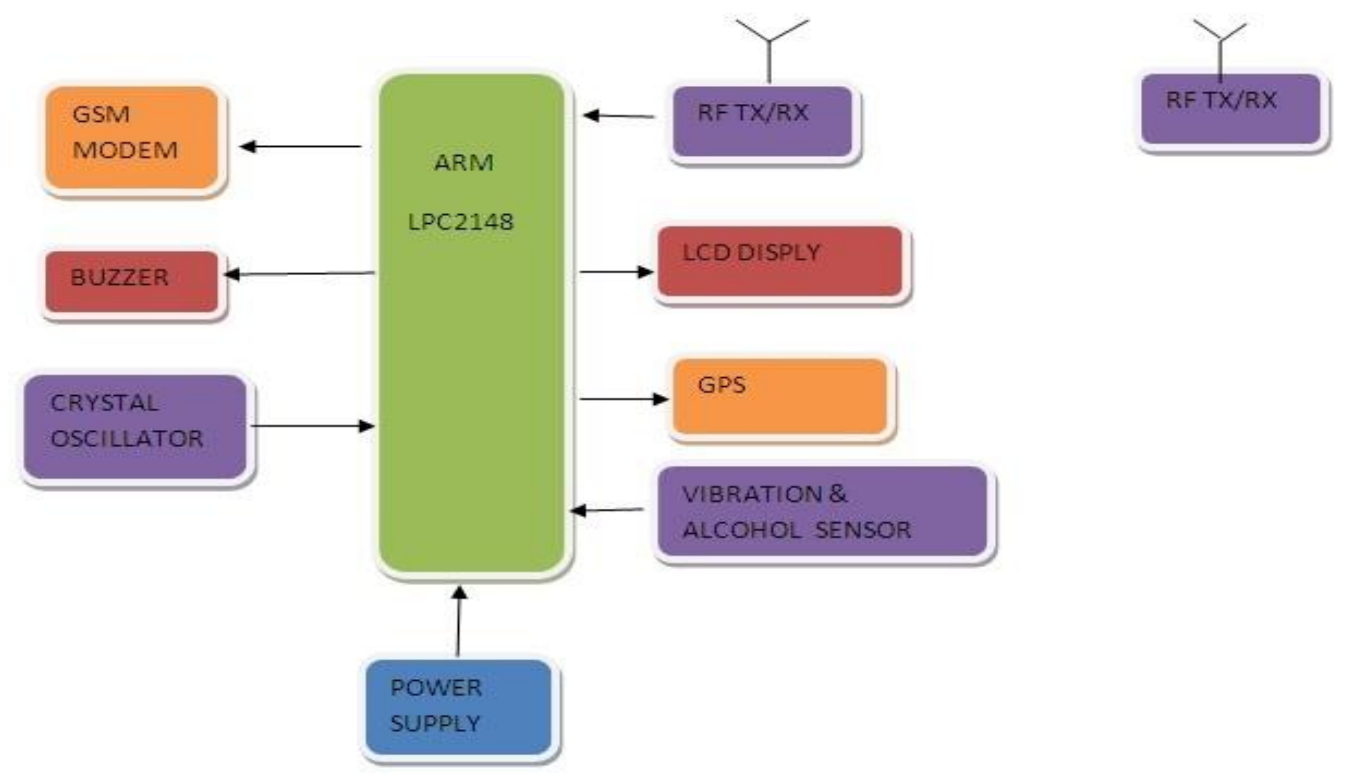

Block diagram [4] of proposed system is as shown in Fig. 2. Here our system can detect the accident using Vibration sensor. Vibration sensor changes its resistance depending. upon amount of bend due to accident. Connected to ARM7 micro-controller. There are threshold values of these sensor outputs. If sensor's output is more than this threshold value then it indicates that accident has occurred. But when the condition of breaking comes it is extremely difficult. System consists on board and road side unit.

\section{Response Time \& Exact Position Of Rsu}

Response time is the total time it takes for a vehicle driver to perceive, evaluate, decide and react to a situation on the roadway. Since vehicle driver response time can be as long as 2-4 seconds, highway designers, accident reconstructionists and the courts take this into account. A highway designer allows several seconds of unobstructed view of a traffic control sign, giving time for the motorist to respond to the sign information.

To fix the position of our RSU unit we must maintain the time which is more than the response time 2-4 seconds. 


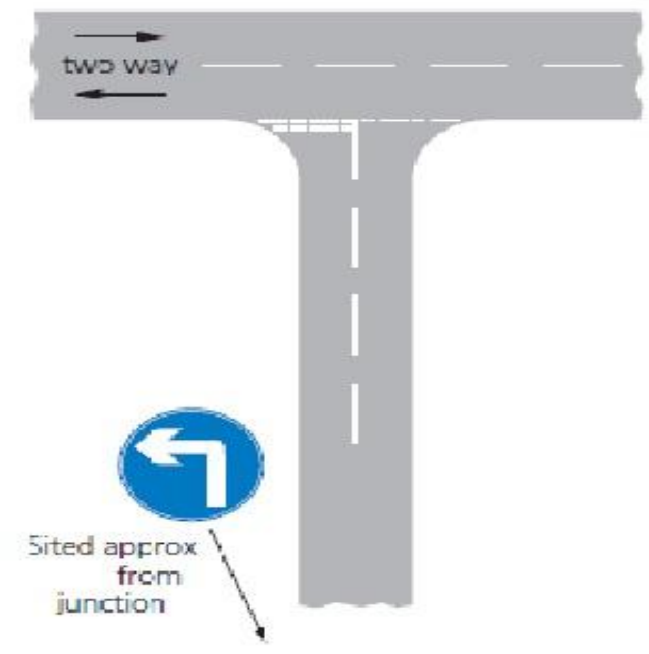

For case: 1

Max speed of vehicle $=120 \mathrm{~km} / \mathrm{hr}$

Speed of vehicle in meter per second $=120 * 5 / 18=33.3333$ meter $/ \mathrm{sec}$

To maintain response time distance between junction and RSU is

Min distance $=2 * 33.33=66.66$ meters... $(2$ is response time $)$

For case: 2

Max speed of vehicle $=180 \mathrm{~km} / \mathrm{hr}$

Speed of vehicle in meter per second $=180 * 5 / 18=50$ meter $/ \mathrm{sec}$

To maintain response time distance between junction and RSU is

Min distance $=2 * 50=100$ meters... ( 2 is response time $)$

So we fix our RSU 100 meters before the junction as shown in fig.

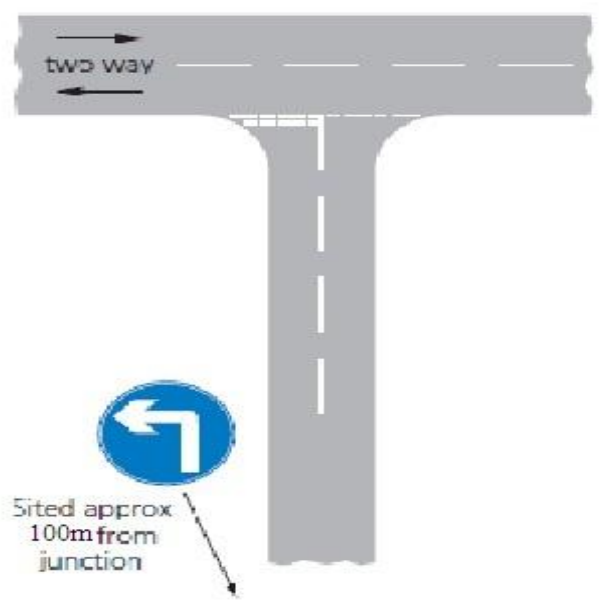

\section{A. Microcontroller}

In this project the controller used is ARM7 LPC2148. LPC2138 CPU module is based on LPC2148 SOC from NXP is an ideal platform for applications which such as Industrial control and monitoring device and any such application which needs migration from 8 bit to 32 bit. This CPU module board supports peripherals such as ADC, SPI, I2C, RTC etc

\section{B. GSM Modem}

A GSM modem is a specialized type of modem which accepts a SIM card, and operates over a subscription to a mobile operator, just like a mobile phone. From the mobile operator perspective, a GSM modem looks just like a mobile phone. A GSM modem can be a dedicated modem device with a serial, USB or 
Bluetooth connection, or it may be a mobile phone that provides GSM modem capabilities. It is used to send an SMS to the contacts of the user about the location of the vehicle. It is beneficial in emergency situations.

\section{GPS Modem}

The Global Positioning System (GPS) is a space based radio-navigation system consisting of a constellation of satellites and a network of ground stations used for monitoring and control. GPS is operated and maintained by the Department of Defense (DOD). The GPS is a constellation of satellites in orbit around the Earth which transmit their positions in space as well as the precise time. It is the receiver that collects data from the satellites and computes its location anywhere in the world based on information it gets from the satellites

GPS data is displayed in different message formats over a serial interface. There are standard and nonstandard (proprietary) message formats. Nearly all GPS receivers output NMEA (National Marine Electronics Association) data. The NMEA standard is formatted in lines of data called sentences. Each sentence contains various bits of data organized in comma delimited format (i.e. data separated by commas). Here are example NMEA sentences from a GPS receiver with satellite lock (4+ satellites, accurate position):

\$GPRMC,235316.000,A,4003.9040,N,10512.5792,W,0.09,144.75,141112,, ${ }^{*} 19$

\$GPGGA,235317.000,4003.9039,N,10512.5793,W,1,08,1.6,1577.9,M,20.7,M,,0000*5F

\$GPGSA,A,3,22,18,21,06,03,09,24,15,,,,,2.5,1.6,1.9*3E

For example, the GPGGA sentence contains the follow:

- Time: 235317.000 is 23:53 and 17.000 seconds in Greenwich mean time

- Longitude: 4003.9040,N is latitude in degrees. decimal minutes, north

- Latitude: 10512.5792,W is longitude in degrees. Decimal minutes, west

- Number of satellites seen: 08

- $\quad$ Altitude: 1577 meters

\section{Alcohol Gas Sensor}

This alcohol sensor is suitable for detecting alcohol concentration on your breath, just like your common breathalyzer. It has a high sensitivity and fast response time. Sensor provides an analog resistive output based on alcohol concentration. The drive circuit is very simple; all it needs is one resistor. A simple interface could be a $0-3.3 \mathrm{~V}$ ADC.

\section{E. RF Module (Rx/Tx)}

The corresponding frequency range varies between $30 \mathrm{kHz}$ and $300 \mathrm{GHz}$. In this RF system, the digital data is represented as variations in the amplitude of carrier wave. This kind of modulation is known as Amplitude Shift Keying (ASK). Transmission through RF is better than IR (infrared) because of many reasons.

\section{Vibration Sensor}

Vibration sensor is used originally as vibration switch because of its high sensitivity; it is sensitive to environment vibration, and generally used to detect the ambient vibration strength. When module did not reach the threshold in shock or vibration strength, DO port output gets high level and when external vibration strength exceeds the threshold, D0 port output gets low level. In vibration sensing, Mass \& Stiffness is important parameter. Mass is represented by an object that wants to move or rotate. Stiffness is represented by springs or constraints of that movement

$\mathrm{fn}=1 / 2 \Pi \sqrt{\mathrm{k}} / \mathrm{m}$

Where: $\mathrm{fn}=$ natural frequency $(\mathrm{Hz}) \mathrm{k}=$ stiffness $(\mathrm{lb} / \mathrm{in}) \mathrm{m}=$ mass mass $=$ weight/gravity weight $(\mathrm{lb})$ gravity (386.1 in/sec 2 ).
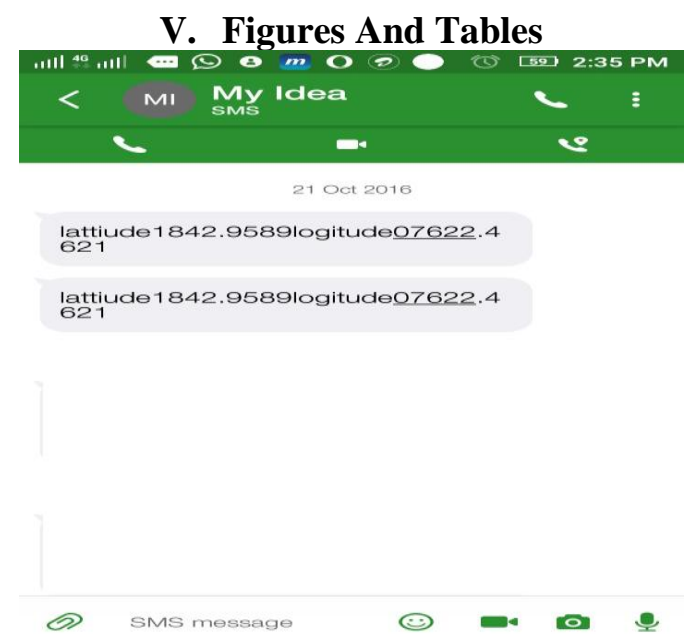


\section{Conclusion}

This paper presents a system for detecting the road side symbols from moving vehicle and a novel approach for Driver Guidance System. The system designed is used to assist driver and is capable to show the symbol details so that the driver can concentrate on driving only. An In-car system is designed with GPS and receivers which is placed inside car. And the road side symbols are built with the transmitters. Thus, the data emitted by transmitters is received by the In-car system and displayed on LCD. The real time implementation of the system will assure safe driving. If further implemented; this system can provide more applications such as text reorganization on the road side boards. This system is very much useful on highways for assisting the driver.

\section{Journal Papers:}

\section{References}

\section{References}

[1]. Bankar Sanket Anil, Kale Aniket Vilas, Prof. S. R. Jagtap," Intelligent System for Vehicular Accident Detection and Notification", 978-1-4799-3358-7114/\$31.00 (C2014 IEEE International Conference on Communication and Signal Processing, April 3-5,2014, India

[2]. Montaser N. Ramadan, Mohammad A. Al-Khedher, Senior Member, IACSIT, and Sharaf A. Al-Kheder "Intelligent Anti-Theft and Tracking System for Automobiles" Vol. 2, No. 1, Feb 2012.

[3]. Pravada P. Wankhade and Prof. S.O. Dahad Government College Of Engineering/Department of Electronics and Telecommunication, Amravati (Maharashtra),India "Real Time Vehicle Locking and Tracking System using GSM and GPS Technology-An Anti-theft System “ Jan-March 2011-Vol.2.No.3.

[4]. Pranav Dhole1, Saba Shaikh2, Nishad Gite3, Vijay Sonawane4, Sandip Institute of Technology and Research Centre, Nashik, India. International Journal of Emerging Trends in Science and Technology,IJETST-Vol.||02||Issue||04||Pages 2285-2288||April||ISSN 2348-9480.

[5]. Vidya Lakshmi, J.R.Balakrishnan, Anand Institute of Higher Technology, Affiliated to Anna University. International Journal of Scientific and Research Publications, Volume 2, Issue 4, April 2012.

[6]. INOOPUR PATNE, 2MANGALA MADANKAR, G.H. Raisoni College of Engineering,Nagpur, India, IRF International Conference, 13th April-2014, Pune, India. 